\title{
40. Über die Existenz der galoisschen Körper mit der vorgegebenen p-Gruppe.
}

Von Tadao Tannaka.

Sendai.

(Comm. by T. TAKagI, M.I.A., May 12, 1936.)

Es ist bekanntlich eine noch heute ungelöste Frage der galoisschen Theorie, ob es über einem festen Grundkörper einen galoisschen Oberkörper mit der vorgegebenen Gruppe gibt. Heutzutage wurde dieses Problem, wenigstens für den speziellen Grundkörper, auch ringtheoretisch behandelbar, und die vollständige Lösung für den Fall, dass die vorgegebene Gruppe eine $p$-Gruppe ist und dass der Grundkörper die Charakteristik $p$ hat, wurde durch Herrn Witt gegeben (Crelles Journal Bd. 174 (1936)). Wie er selbst in seiner Arbeit erkannt hat, ist das Konstruktionsproblem auch beim Zahlkörper, wenn der Grundkörper die $p$-te Einheitswurzeln enthält, auf das Zerfallen der gewissen Algebren reduziert. Man kann durch seine Konstruktionsmethode ziemlich einfach den folgenden Satz beweisen:

Satz: Wenn der Grundkörper $k$ die p-te Einheitswurzeln ( $p$ eine Primzahl) enthält, so gibt es über $k$ unendlichviele Erweiterungskörper mit beliebig vorgegebener p-Gruppe als galoissche Gruppe.

Eine ausführliche Darstellung soll in einem anderen Orte erscheinen. 